Génét. Sél. Evol., 1984, 16 (4), 445-454

\title{
Estimation de la valeur génétique des reproducteurs dans les cas d'incertitude sur les apparentements I. Formulation des indices de sélection
}

\author{
J.P. POIVEY et J.M. ELSEN \\ I.N.R.A., Station d'Amélioration génétique des Animaux, \\ B.P. 12, F 31320 Castanet-Tolosan
}

\begin{abstract}
Résumé
Quand les accouplements ne sont pas contrôlés, il peut y avoir des incertitudes sur les paternités et il faut en tenir compte dans les indices de sélection. On donne ici l'expression du critère de sélection qui maximise le gain génétique dans ces situations (l'espérance conditionnelle). On lui compare la régression linéaire qui est beaucoup plus facile à utiliser, mais dont on ne sait pas si elle permet de faire des progrès importants. Quelques simulations montrent que ce deuxième critère est utilisable sans diminution sensible de l'espérance du progrès génétique, par rapport à l'espérance conditionnelle.
\end{abstract}

Mots clés: Indexation, erreurs de filiation.

\begin{abstract}
Summary
Prediction of genetic merit in the case of uncertain paternity. I. Deriving selection indices
\end{abstract}

When mating is not controlled, paternity may be wrongly attributed in some cases and such errors have to be taken into account within selection indices. Formulae for the selection criterion which maximizes genetic gain (i.e. for the conditional expectation) in such situations are given. This criterion is compared to linear regression, which is much easier to compute but does not guarantee the largest gains. Some simulations show that linear regression may be applied without any perceptible decrease in the expectation of genetic progress as compared to the use of the conditional expectation.

Key words : Selection index, uncertain paternity.

\section{Introduction}

L'estimation de la valeur génétique d'un animal candidat à la sélection s'effectue à partir de l'information disponible concernant ses propres performances phénotypiques et celles des individus qui lui sont apparentés. Dans de nombreux schémas de sélection, 
les fécondations sont rigoureusement contrôlées, souvent grâce à la pratique de l'insémination artificielle, de sorte que les parentés y apparaissent comme des données exactes. Cependant, dans un certain nombre de situations, en particulier quand on utilise la monte naturelle, on ne peut pas connaître les accouplements avec certitude.

Le problème se pose d'une façon tout à fait générale dans des milieux d'élevage traditionnels, comme en Afrique où les parentés dans les troupeaux peuvent être définies comme un ensemble d'événements aléatoires.

S'intéressant plus particulièrement aux ovins, BoNAITI (1979) a chiffré, en terme de précision de l'indexation, la perte d'efficacité due à ces erreurs quand les valeurs génétiques des reproducteurs sont estimées classiquement sous l'hypothèse de certitudes sur les filiations. Une autre démarche, que nous développerons ici, consiste à tenir compte explicitement de ces incertitudes dans la formulation des indices de sélection. L'objectif est donc d'utiliser pour le choix des reproducteurs, une quantité maximale d'informations sur les liens de parenté possibles.

Le premier problème est alors de déterminer la loi de probabilité de ces liens de parenté. Dans cette première partie nous n'aborderons pas les méthodes pratiques de détermination de ces probabilités, mais étudierons, d'un point de vue théorique, l'expression analytique de critères de sélection utilisables dans ces situations d'incertitude. Une deuxième partie sera consacrée à l'application de ces critères au cas où les probabilités de filiation sont déterminées à partir de la comparaison des dates de mises-bas et de fécondation supposées.

\section{Définitions et hypothèses}

On s'intéresse à un groupe de m mâles $\left(A_{1}, A_{2} \ldots A_{i} \ldots A_{m}\right)$ dont on veut évaluer la valeur génétique par un testage sur descendance. On dispose pour ce faire d'une information mesurée sur $N$ descendants $\left(D_{1}, D_{2} \ldots D_{j} \ldots D_{N}\right)$ sans que les filiations soient connues avec certitude. On suppose cependant qu'aucun lien de parenté n'existe entre les parents de ces $\mathrm{N}$ individus, qu'il s'agisse des $\mathrm{m}$ pères que l'on veut tester, des femelles auxquelles ils ont été accouplés ou des éventuels mâles extérieurs au groupe et ayant fécondé accidentellement ces mères. Il existe un nombre fini de situations possibles entre les $A_{i}$ et les $D_{j}$ et nous faisons l'hypothèse que l'on peut attribuer à chacune de ces situations une probabilité de réalisation a priori, indépendamment de l'observation des performances.

Plus précisément, une situation globale de parenté $S_{k}$, sera définie par l'ensemble des liens de parenté entre tout couple d'individus de la population formée des $\mathrm{m}$ pères et $\mathbf{N}$ descendants ; alors qu'une situation élémentaire de parenté décrira la relation de filiation entre un père $A_{i}$ et un descendant $D_{j}$ particuliers : $D_{j}$ est ou n'est pas fils de $A_{i}$ (on notera $j$ (i) l'événement « $D_{j}$ est fils de $A_{i}$ »). Une situation globale de parenté est alors la réunion de $m . N$ situations élémentaires de parenté.

Nous distinguerons donc parmi les probabilités a priori les probabilités globales $P\left(S_{k}\right)$ des probabilités élémentaires $P(j(i))$. L'ensemble des situations globales forme un système complet d'événements : $\sum_{k} P\left(S_{k}\right)=1$. Nous noterons $\Omega$ leur loi de probabilité, entièrement déterminée par la donnée des $\mathrm{P}\left(\mathrm{S}_{\mathrm{k}}\right)$. 
Remarquons par ailleurs que la probabilité de l'événement j (i) conditionné par la réalisation de la structure $S_{k}, P\left(j(i) / S_{k}\right)$, est 1 ou 0 , selon que dans $S_{k}, D_{j}$ est ou non fils de $A_{i}$.

Ces probabilités a priori sont données par les informations dont on dispose sur les apparentements possibles. Ainsi, le mode de conduite et le plan d'accouplement pratiqués fournissent des renseignements sur ces probabilités : si les femelles sont regroupées en lots de testage ( $\mathrm{L}_{\mathrm{i}}$ étant le lot de femelles supposées accouplées au mâle $\mathrm{A}_{\mathrm{i}}$ ) on excluera peut-être toute paternité possible entre les mâles du troupeau autres que $A_{i}$ et les descendants du lot $\mathrm{L}_{\mathrm{i}}$, tout en envisageant une paternité extérieure due à une introduction accidentelle d'un mâle étranger. Si les femelles sont synchronisées, on supposera peut-être que tous ces accouplements accidentels sont le fait d'un même mâle. Au contraire, dans le cas d'une reproduction continue dans le temps, l'hypothèse de non-apparentement entre ces fils de pères extérieurs sera acceptable. La comparaison des dates de mises à la reproduction à celles des mises-bas donnent également des informations utilisables pour estimer ces probabilités a priori quand les femelles sont saillies en lots successifs. L'observation de marqueurs génétiques, comme les gènes de coloration ou les groupes sanguins, est aussi un outil efficace de ce point de vue.

Notons enfin que l'indépendance ou la non-indépendance des situations élémentaires de parenté dépend de la biologie de l'espèce. Si les reproducteurs ont peu de descendants chacun, la réalisation de l'événement « $D_{j 1}$ est fils de $A_{i}$ " ne saurait être considérée comme indépendante du fait que $A_{i}$ ait déjà un autre fils $\left(D_{j 2}\right)$ :

$$
P(j 1(i) / j 2(i)) \neq P(j 1(i))
$$

Au contraire, si le nombre potentiel de fils par père est grand, l'indépendance entre situations élémentaires est une bonne approximation. Remarquons que, dans ce cas, la probabilité globale $P\left(S_{k}\right)$ est le produit des $N$ probabilités élémentaires $P(j(i))$ correspondant aux événements élémentaires $j$ (i) réalisés dans $S_{k}$.

\section{Résultats}

\section{A. Utilisation de l'espérance conditionnelle comme indice de sélection}

Soit $\mathrm{Y}$ le $\mathrm{m}$-vecteur ligne des valeurs génétiques des pères et $\mathrm{X}$ le $\mathrm{N}$-vecteur ligne des performances mesurées chez les descendants. Nous supposerons que les lois conjointes de $\mathrm{Y}$ et de $\mathrm{X}$, conditionnées par la réalisation d'une situation globale $\mathrm{S}_{k}$ sont multinormales. Si les moments de ces lois et la loi de probabilité $\Omega$ des situations de parenté sont connus, on peut utiliser l'espérance conditionnelle comme critère de classification des pères. En effet, Cochran (1951) a montré que, quand on retient tout individu dont l'indice dépasse un seuil $\lambda_{\alpha}$ fixé par le taux de sélection $\alpha$, on maximise l'espérance du progrès génétique ( $\lambda_{\alpha}$ est tel, qu'en espérance, une fraction $\alpha$ des candidats est retenue). Ce résultat fut obtenu dans le cas particulier où l'ensemble des informations (X) se répartit en $\mathrm{m}$ groupes indépendants les uns des autres et tels que tous les candidats sont connus avec la même précision : $Y_{1}$ est estimé à partir de $X_{11}, X_{12} \ldots, X_{1 n}$; $\mathrm{Y}_{2}$ à partir de $\mathrm{X}_{21}, \mathrm{X}_{22} \ldots \mathrm{X}_{2 \mathrm{n}}$, etc., avec :

$$
\begin{aligned}
& \operatorname{cov}\left(Y_{i}, X_{i j}\right)=\operatorname{cov}\left(Y_{i^{\prime}}, X_{i^{\prime} j}\right) \\
& \operatorname{cov}\left(Y_{i}, X_{i^{\prime} j}\right)=0 \\
& \operatorname{cov}\left(X_{i j}, X_{i j^{\prime}}\right)=\operatorname{cov}\left(X_{i^{\prime} j}, X_{i^{\prime} j^{\prime}}\right)
\end{aligned}
$$


GOFFINET \& ELSEN (1984) ont généralisé ce résultat au cas où l'on a sur les candidats à la sélection des quantités d'informations inégales; aléatoires et éventuellement, non indépendantes, ce qui est la situation qui nous intéresse ici.

Ils ont montré notamment que le seuil optimal au-delà duquel les pères sont retenus est unique $\left(\lambda_{\alpha_{i}}=\lambda_{\alpha}\right.$, quel que soit $\left.i\right)$.

L'espérance conditionnelle s'écrit :

$$
\mathrm{E}(\hat{\mathrm{Y}} / \mathrm{X})=\sum_{\mathrm{k}} \mathrm{P}\left(\mathrm{S}_{\mathrm{k}} / \mathrm{X}\right) \cdot \mathrm{E}\left(\mathrm{Y} / \mathrm{X} \text { et } \mathrm{S}_{\mathrm{k}}\right)
$$
$\operatorname{des} \hat{\mathbf{y}}_{\mathrm{i}}$.

Nous noterons $\hat{y}$ une réalisation (pour $X=x$ ) de la variable $\hat{Y}, \hat{y}$ étant le $\mathrm{m}$ vecteur

Sous l'hypothèse de normalité :

$$
\mathrm{E}\left(\mathrm{Y} / \mathrm{X}=\mathrm{x} \text { et } \mathrm{S}_{\mathrm{k}}\right)=\mu_{\mathrm{yk}}+\mathrm{C}_{\mathrm{k}} \cdot \mathrm{V}_{\mathrm{k}}^{-1}\left(\mathrm{x}-\mu_{\mathrm{xk}}\right)^{\prime}
$$

où - $\mu_{\mathrm{xk}}$ et $\mu_{\mathrm{yk}}$ sont les vecteurs des espérances de $\mathrm{X}$ et $\mathrm{Y}$;

- $\mathrm{C}_{\mathrm{k}}$ et $\mathrm{V}_{\mathrm{k}}$ les matrices des covariances entre les $\mathrm{Y}$ et les $\mathrm{X}$ et des covariances entre les $\mathrm{X}$, conditionnées par la réalisation de $\mathrm{S}_{\mathrm{k}}$;

- $\left(\mathrm{x}-\mu_{\mathrm{xk}}\right)^{\prime}$ est le vecteur colonne transposé de $\left(\mathrm{x}-\mu_{\mathrm{xk}}\right)$.

La probabilité $P\left(S_{k} / X=x\right)$ de la réalisation de la situation $S_{k}$ quand on connaît la valeur $\mathrm{x}$ des prédicteurs peut s'écrire :

$$
P\left(S_{k} / X=x\right)=\frac{f_{k}(x) \cdot P\left(S_{k}\right)}{\sum_{h} f_{h}(x) \cdot P\left(S_{h}\right)}
$$

$\mathrm{f}_{\mathrm{h}}$ étant la densité de la loi de $\mathrm{X}$ conditionnelle à la situation $\mathrm{S}_{\mathrm{h}}$. Alors,

$$
\hat{y}=\mu_{y k}+\frac{\sum_{k} P\left(S_{k}\right) \cdot f_{k}(x) \cdot C_{k} \cdot V_{k}^{-1} \cdot\left(x-\mu_{x k}\right)^{\prime}}{\sum_{h} P\left(S_{h}\right) \cdot f_{h}(x)}
$$

Par la suite, nous supposerons les variables centrées $\left(\mu_{\mathrm{xk}}=0, \mu_{\mathrm{yk}}=0\right)$.

Dans le cas où il n'y a qu'un descendant par mère (sélection sur des familles de demi-frères), et en supposant l'absence de dominance et d'épistasie ainsi que la nonconsanguinité des parents, on a :

$$
\begin{aligned}
& \operatorname{cov}\left(\left(Y_{i}, X_{j}\right) / S_{k}\right)=\frac{1}{2} \sigma_{a}^{2} \text { si } j \text { est fils de } i\left(\sigma_{a}^{2} \text { étant la variance génétique additive }\right) . \\
& \begin{aligned}
& \operatorname{cov}\left(\left(Y_{j}, X_{j}\right) / S_{k}\right)= 0 \text { sinon } \\
& \text { et } \operatorname{cov}\left(\left(X_{j}, X_{j^{\prime}}\right) / S_{k}\right)= \sigma_{\mathrm{p}}^{2} \text { si } j=j^{\prime} \\
& \frac{1}{4} \sigma_{a}^{2} \text { si } j \text { et } j^{\prime} \text { sont demi-frères } \\
& 0 \text { sinon. }
\end{aligned}
\end{aligned}
$$

Sous ces hypothèses, le ième élément du vecteur $C_{k} V_{k}^{-1} x^{\prime}$, correspondant au père $A_{i}$, peut s'écrire :

$$
\frac{1 / 2 n_{i k} h^{2}}{1+1 / 4\left(n_{i k}-1\right) h^{2}} x_{i k}
$$

$\mathrm{n}_{\mathrm{ik}}$ étant le nombre de fils de $A_{\mathrm{i}}$ dans la situation $\mathrm{S}_{\mathrm{k}}, \mathrm{x}_{\mathrm{ik}}$, leur valeur moyenne et $\mathrm{h}^{2}$ l'héritabilité $\sigma_{\mathrm{a}}^{2} / \sigma_{\mathrm{p}}^{2}$ 
L'espérance conditionnelle

$$
\hat{y}_{i}=\frac{\sum_{k} P\left(S_{k}\right) \cdot f_{k}(x) \cdot \frac{1 / 2 n_{i k} h^{2}}{1+1 / 4\left(n_{i k}-1\right) h^{2}} \cdot x_{i k}}{\sum_{h} P\left(S_{h}\right) \cdot f_{h}(x)}
$$

Ce n'est donc pas un critère de formulation simple, car faisant intervenir $S_{k}$, et on est amené à chercher des critères plus faciles à mettre en œuvre. Notons auparavant qu'avec ce critère l'espérance du progrès génétique obtenu en appliquant la règle de COCHRAN, serait :

$$
\begin{aligned}
& \left.\hat{\Lambda}=\frac{1}{\alpha} \sum_{i=1} \sum_{k} P / S_{k}\right)\left(S_{k}\right) \int_{\lambda_{\alpha}}^{\infty} \hat{y}_{i} \cdot \phi_{k}\left(\hat{Y}_{i}\right) \cdot d \hat{y}_{i} \\
& \alpha=\frac{1}{m} \cdot \sum_{i=1}^{m} \sum_{k} P\left(S_{k}\right) \int_{\lambda_{\alpha}}^{\infty} \phi_{k}\left(\hat{Y}_{i}\right) \cdot d \hat{y}_{i}
\end{aligned}
$$

où $\phi_{\mathrm{k}}$ est la densité conditionnelle de $\hat{\mathrm{Y}}$ à la réalisation de $\mathrm{S}_{\mathrm{k}}$. La recherche du seuil de sélection est donc en pratique irréalisable lorsque l'on utilise un tel critère.

\section{B. Utilisation de la régression linéaire comme indice de sélection}

Si on se limite à la classe des prédicteurs linéaires en $\mathrm{x}$, on est tenté de choisir la régression linéaire que l'on sait être, dans cette classe, le meilleur critère au sens des moindres carrés.

Sous les mêmes hypothèses que précédemment nous classerions donc les pères sur le vecteur d'indices de sélection $\tilde{y}$ tel que :

$$
\tilde{y}=C \cdot V^{-1} \cdot x^{\prime}
$$

où $\mathrm{C}$ et $\mathrm{V}$ sont les matrices de covariances entre les $\mathrm{Y}$ et les $\mathrm{X}$ et entre les $\mathrm{X}$, donc telles que :

$$
\begin{aligned}
& \mathrm{C}=\sum_{k} \mathrm{P}\left(\mathrm{S}_{\mathrm{k}}\right) \cdot \mathrm{C}_{\mathrm{k}} \\
& \mathrm{V}=\sum_{\mathrm{k}} \mathrm{P}\left(\mathrm{S}_{\mathrm{k}}\right) \cdot \mathrm{V}_{\mathrm{k}}
\end{aligned}
$$

On trouve que :

$$
\begin{gathered}
\operatorname{cov}\left(\mathrm{Y}_{\mathrm{i}}, \mathrm{X}_{\mathrm{j}}\right)=\mathrm{P}[\mathrm{j}(\mathrm{i})] \cdot 1 / 2 \cdot \sigma_{\mathrm{a}}^{2} \\
\operatorname{var}\left(\mathrm{X}_{\mathrm{j}}\right)=\sigma_{\mathrm{p}}^{2} \\
\operatorname{cov}\left(\mathrm{X}_{\mathrm{j}}, \mathrm{X}_{\mathrm{j}^{\prime}}\right)=\left(\sum_{\mathrm{i}=1}^{\mathrm{m}} \mathrm{P}[\mathrm{j}(\mathrm{i})] \cdot \mathrm{P}\left[\mathrm{j}^{\prime}(\mathrm{i}) / \mathrm{j}(\mathrm{i})\right] \cdot 1 / 4 . \sigma_{\mathrm{a}}^{2}\right)
\end{gathered}
$$

Si la règle de sélection est toujours de retenir le mâle $A_{i}$ quand son indice $\tilde{y}_{i}$ est supérieur à un seuil $\lambda_{\alpha_{\mathrm{i}}}$ (sans supposer ici que les seuils sont égaux), on peut calculer l'espérance de la valeur génétique de $A_{i}$ quand il est sélectionné :

$$
E\left(Y_{i} / \bar{y}_{i}>\lambda_{\alpha_{i}}\right)=\frac{\int_{\lambda_{\alpha_{i}}}^{\infty} d \tilde{y}_{i} \int_{-\infty}^{\infty} y_{i} g\left(y_{i}, \tilde{y}_{i}\right) d y_{i}}{\int_{\lambda_{\alpha_{j}}}^{\infty} d \tilde{y}_{i} \int_{-\infty}^{\infty} g\left(y_{i}, \tilde{y}_{i}\right) d y_{i}}
$$


où $\mathrm{g}$ est la loi conjointe de la valeur génétique $\mathrm{Y}_{\mathrm{i}}$ et de son prédicteur $\tilde{\mathrm{Y}}_{\mathrm{i}}$ :

En effet, $g\left(y_{i}, \tilde{y}_{i}\right)=\sum_{k} P\left(S_{k}\right) \cdot g_{k}\left(y_{i}, \tilde{y}_{i}\right)$ et la loi conditionnée par la réalisation de $S_{k}, g_{k}$, est, sous nos hypothèses, binormale, d'espérance nulle et de variance :

$$
\left(\begin{array}{ll}
\sigma_{\mathrm{a}}^{2} & ; \mathrm{C}_{\mathrm{ki}} \mathrm{V}^{-1} \mathrm{C}_{\mathrm{i}}^{\prime} \\
\mathrm{C}_{\mathrm{j}} \mathrm{V}^{-1} \mathrm{C}_{\mathrm{ki}}^{\prime} ; & \mathrm{C}_{\mathrm{i}} \mathrm{V}^{-1} \mathrm{~V}_{\mathrm{k}} \mathrm{V}^{-1} \mathrm{C}_{\mathrm{i}}^{\prime}
\end{array}\right)
$$

$C_{i}$ et $C_{k i}$ étant les ièmes lignes des matrices $C$ et $C_{k}$.

Alors, si $\alpha_{k i}$ est la probabilité que le mâle $A_{i}$ soit retenu quand $S_{k}$ est réalisé et $\mathrm{Z}_{\mathrm{ki}}$ la valeur de la densité $\tilde{\mathrm{Y}}_{\mathrm{i}}$ au point de troncature

$$
E\left(Y_{i} / \tilde{Y}_{i}>\lambda_{\alpha_{j}}\right)=\frac{\sum_{k} P\left(S_{k}\right) \cdot z_{k i} \cdot C_{k i} \cdot V^{-1} \cdot C_{i}^{\prime}}{\sum_{k} P\left(S_{k}\right) \cdot \alpha_{k i}}
$$

avec $\alpha_{\mathrm{ki}}=\int_{-\infty}^{\infty} \mathrm{dy}_{\mathrm{i}} \int_{\lambda_{\alpha_{i}}}^{\infty} \mathrm{g}_{\mathrm{k}}\left(\mathrm{y}_{\mathrm{i}}, \bar{y}_{\mathrm{i}}\right) \mathrm{dy} \tilde{y}_{\mathrm{i}}$
et $\mathrm{z}_{\mathrm{ki}}=\frac{1}{\sqrt{2 \Pi \mathrm{C}_{\mathrm{i}} \mathrm{V}^{-1} \mathrm{~V}_{\mathrm{k}} \mathrm{V}^{-1} \mathrm{C}_{\mathrm{i}}^{\prime}}} \exp \left(-\frac{1}{2} \cdot \frac{\lambda_{\alpha_{\mathrm{i}}}^{2}}{\mathrm{C}_{\mathrm{i}} \mathrm{V}^{-1} \mathrm{~V}_{\mathrm{k}} \mathrm{V}^{-1} \mathrm{C}_{\mathrm{i}}^{\prime}}\right)$

L'espérance $\bar{\Lambda}$ du progrès génétique est donc :

$$
\bar{\Lambda}=\frac{1}{\alpha} \sum_{i=1}^{m}\left(\sum_{k} P\left(S_{k}\right) \cdot z_{i k} \cdot C_{k i} V^{-1} C_{i}^{\prime}\right)
$$

mais ici, contrairement à l'espérance conditionnelle, les seuils optimaux $\lambda_{\alpha_{i}}$ ne sont pas nécessairement les mêmes d'un père à l'autre (on peut le voir en maximisant $\bar{\Lambda}$ avec la contrainte :

$$
\left.\sum_{i=1}^{m} \sum_{k} P\left(S_{k}\right) \cdot \alpha_{k i}=m \alpha\right)
$$

En outre, il n'y a aucune raison pour que la régression linéaire soit le meilleur indice de sélection, au sens de la maximisation du progrès génétique, dans la classe des critères linéaires. Ce meilleur critère peut, en théorie, être déterminé à partir de l'expression du progrès génétique. En pratique, cette approche ne donne pas de résultats simples, et nous l'avons abandonnée.

En conclusion, on a donc le choix entre un premier critère, l'espérance conditionnelle qui fournit le progrès génétique maximum, mais qui est généralement inutilisable du fait de sa complexité et un second, la régression linéaire, simple à calculer mais qui n'assure pas nécessairement le progrès maximum. Si, toutefois, le gain obtenu se révélait ne pas en être trop éloigné, il serait certainement préférable d'adopter ce critère. Malheureusement, dans le cas général, les expressions des progrès $(\hat{\Lambda}$ et $\hat{\Lambda})$ sont elles aussi très complexes et il n'y a pas de réponse simple au problème.

Dès lors, une bonne démarche pourrait être, d'une part de raisonner chaque situation particulière compte tenu des simplifications éventuelles qui lui sont propres, d'autre part, d'utiliser les techniques de simulation pour estimer et comparer les espérances de progrès génétique. 


\section{Exemple d'application}

\section{A. Présentation de l'étude}

Nous nous plaçons maintenant dans la situation particulière où les $\mathrm{N}$ descendants peuvent être regroupés en $m$ groupes, éventuellement chevauchants (un même descendant peut appartenir simultanément à plusieurs groupes). Nous définirons ces groupes par le fait que tous les individus ayant une probabilité non nulle d'être fils d'un même père $A_{i}$ appartiennent à un même groupe $G_{i}$.

Concrètement, cette situation peut se rencontrer en diverses occasions déjà évoquées. Notons tout d'abord que, quand les groupes sont entièrement chevauchants (tous confondus et formés de la totalité des $\mathrm{N}$ descendants), on est dans la situation générale présentée dans le premier paragraphe. Deux cas moins triviaux peuvent se rencontrer :

- reproduction en séquence où l'utilisation des mâles est répartie dans le temps en périodes successives telles qu'un seul père se reproduit au cours d'une période. Dans cette situation seuls les animaux nés entre 2 dates extrêmes $t_{i}$ et $t_{i+1}$ (correspondant aux limites de la durée de gestation) sont susceptibles d'être fils du père $A_{i}$ mais peuvent aussi être fils des pères $A_{i-1}$ et $A_{i+1}$ qui ont été mis à la reproduction au cours des périodes précédant et suivant celle de $A_{i}$. Nous réservons à une $2^{e}$ publication l'étude détaillée de cette situation ;

- testage sur descendance en ferme avec allotement des reproducteurs (ovins), mais possibilité de saillies accidentelles par des mâles inconnus. Les femelles sont regroupées en autant de lots de conjoints supposés que de mâles à tester, les descendants du ième lot sont, soit fils du père $A_{i}$, soit fils d'un ou plusieurs mâles inconnus.

Dans ce cas nous allons restreindre encore notre champ d'analyse en supposant d'une part, l'indépendance entre situations élémentaires (Pr $[\mathrm{j}(\mathrm{i})$ et $\left.\left.\mathrm{j}^{\prime}\left(\mathrm{i}^{\prime}\right)\right]=\operatorname{Pr}[\mathrm{j}(\mathrm{i})] . \operatorname{Pr}\left[\mathrm{j}^{\prime}\left(\mathrm{i}^{\prime}\right)\right]\right)$ d'autre part, l'équiprobabilité des filiations intra lot $\left(\operatorname{Pr}[j(i)]=p_{i}\right.$ dans le lot $L_{i}, 0$ sinon, pour $\left.i=1,2, \ldots m\right)$. En pratique, ces probabilités $\mathrm{p}_{\mathrm{i}}$ pourront être estimées a priori par sondage en utilisant, par exemple, les contrôles de groupes sanguins et on se contentera d'estimation globale sur chaque élevage, voire sur toute la population $\left(\mathrm{p}_{\mathrm{i}}=\mathrm{p}\right)$.

Quand la population des descendants peut être regroupée en de tels lots de testage, seules les performances $x_{i j}$ des membres du lot $L_{i}$ sont utilisées pour estimer la valeur génétique du père $A_{i}$. Par ailleurs, nous nous placerons dans le cas où ceux de ces individus qui ne sont pas fils de $A_{i}$ sont non apparentés entre eux (fils d'une infinité de pères inconnus). Dans ces conditions, la régression linéaire s'écrit simplement :

$$
\bar{Y}_{i}=\frac{1 / 2 p_{i} h^{2}}{1+1 / 4\left(N_{i}-1\right) p_{i}^{2} h^{2}}\left(\sum_{j=1}^{N_{i}} x_{i j}\right)
$$

alors que, dans l'expression de l'espérance conditionnelle $\hat{Y}_{i}$, la probabilité de réalisation d'une situation $S_{k}$ est $\left(n_{i k}\right.$ étant le nombre de fils de $A_{i}$ dans $\left.S_{k}\right)$ :

$$
p_{i}^{n_{i k}} \cdot\left(1-p_{i}\right)^{N_{i}-n_{i k}}
$$




\section{B. Simulation de la sélection}

La règle de Cochran (1951) (retenir tout candidat dont la valeur de l'indice dépasse un seuil $\lambda_{\alpha}$ ) est, nous l'avons dit, difficilement applicable dans le cas de la régression linéaire puisqu'en toute rigueur, le seuil $\lambda_{\alpha_{i}}$ varie dans ce cas d'un candidat à l'autre. Pour comparer plus simplement régression linéaire et espérance conditionnelle, nous nous sommes placés ici dans le cas où l'on s'impose de retenir, parmi les $\mathrm{m}$ candidats, un nombre de pères $\mathrm{r}$ fixé a priori (les $\mathrm{r}$ meilleurs classés).

Dans ces conditions, nous avons simulé des opérations de sélection, selon le processus décrit figure 1 pour diverses valeurs du nombre de candidats $m$ et de retenus $\mathbf{r}$ (1 à $\mathrm{m}$ ), de l'effectif $\mathrm{N}_{\mathrm{i}}$ de descendants mesurés par père, de l'héritabilité et des probabilités de filiations $\mathrm{p}_{\mathrm{i}}$ (tabl. 1). Dans aucun des cas étudiés l'espérance du progrès génétique par utilisation de la régression linéaire ne s'est montrée inférieure à 0,9 p. 100 de celle qui eût été obtenue avec l'espérance conditionnelle (tabl. 1).

Bien que pour des raisons de temps de calcul, les situations étudiées aient été en nombre restreint et uniquement des cas équilibrés $\left(N_{i}=N_{i}^{\prime}\right.$ et $p_{i}=p_{i}^{\prime}$ quels que soient i et $i^{\prime}$ ), il nous semble donc que l'on puisse préconiser l'utilisation du critère linéaire sans crainte de perte sensible.

TABLEAU 1

Cas étudiés et résultats.

Cases studied and results.

\begin{tabular}{c|c|c|c|c|c}
\hline \hline $\begin{array}{c}\text { Nombre } \\
\text { de pères (m) }\end{array}$ & $\begin{array}{c}\text { Nombre } \\
\text { de filles (N) }\end{array}$ & $\begin{array}{c}\text { Probabilité } \\
\text { de filiation }(\mathrm{p})\end{array}$ & $\begin{array}{c}\text { Héritabilité } \\
\left(\mathrm{h}^{2}\right)\end{array}$ & $\begin{array}{c}\text { Nombre } \\
\text { de simulations } \\
(\mathrm{NSIM})\end{array}$ & $\begin{array}{c}\text { Réduction } \\
\text { de progrès } \\
\text { génétique* } \\
(\mathrm{p} .100)\end{array}$ \\
\hline & 3 & 0,2 & 0,3 & 10000 & 0,9 \\
5 & 3 & 0,2 & 0,6 & 18000 & 0,3 \\
5 & 3 & 0,5 & 0,2 & 9000 & 0,6 \\
5 & 3 & 0,5 & 0,3 & 16000 & 0,1 \\
5 & 3 & 0,8 & 0,3 & 16000 & 0,2 \\
5 & 10 & 0,5 & 0,3 & 3000 & 0,5 \\
5 & 10 & 0,5 & 0,5 & 2500 & 1,7 \\
10 & 3 & 0,5 & 0,3 & 5000 & 0,5 \\
50 & & & 0,5 & \\
\hline
\end{tabular}

* Perte relative due à la sélection sur le critère linéaire par rapport à la sélection sur l'espérance conditionnelle. 


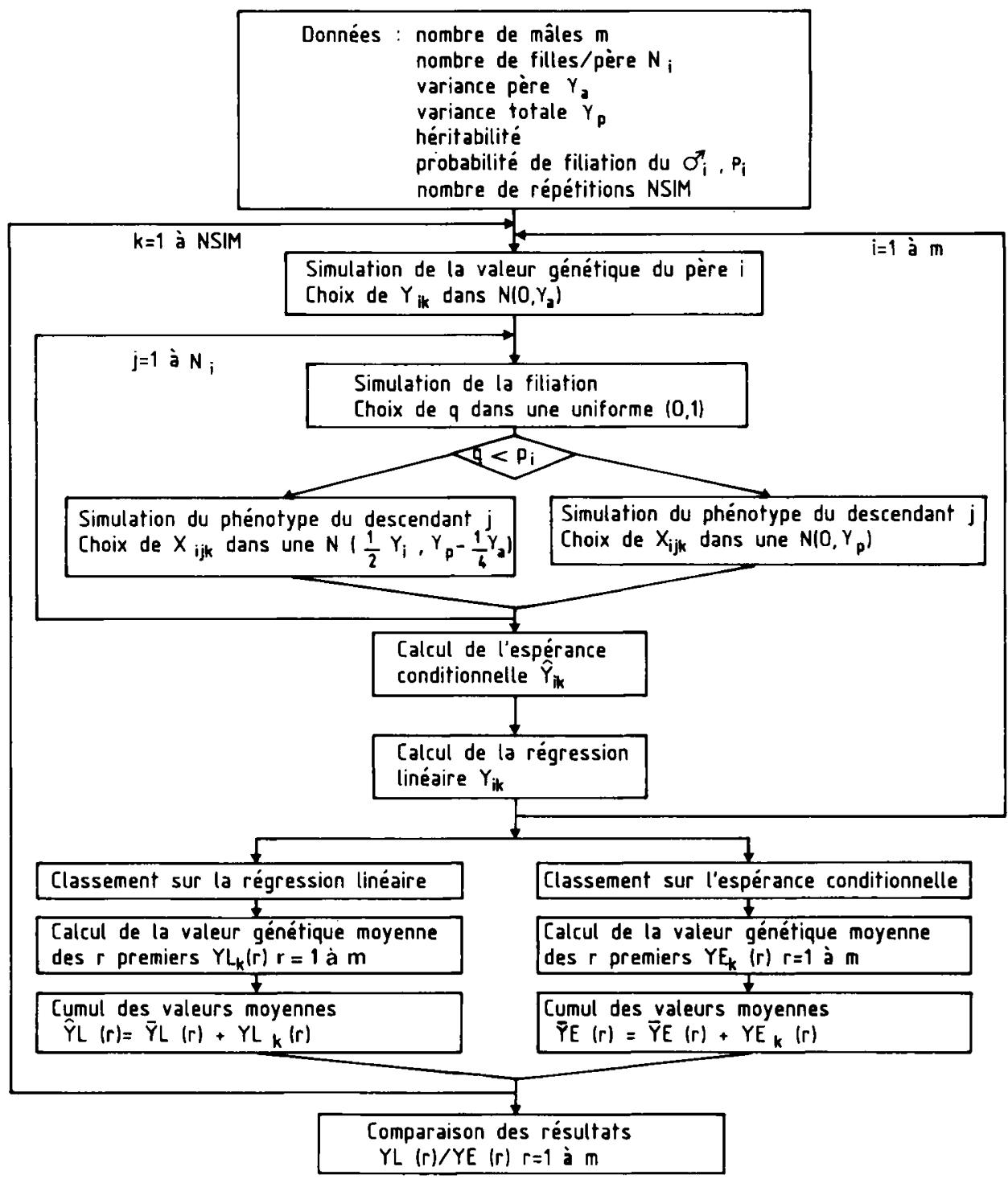

FIG. 1

Simulation des opérations de sélection : méthode utilisée.

Simulation of selection procedure. 


\section{Discussion - Conclusion}

L'application rigoureuse des règles optimales de sélection n'étant pas possible pratiquement dans les cas d'incertitude sur les filiations, on peut espérer, à l'instar de l'exemple développé ici, que l'utilisation de prédicteurs linéaires donne des résultats presque équivalents mais beaucoup plus simplement. Il conviendra de le vérifier cas par cas.

Seule la régression linéaire a été comparée ici à l'espérance conditionnelle. On aurait aussi pu, comme BonAITI (1975), étudier l'efficacité d'un classement des candidats sur l'expression de l'espérance conditionnelle obtenue dans le cas de certitude sur les filiations (dans le cas de la loi normale, c'est aussi la régression linéaire $\bar{Y}_{\mathrm{i}}$ ) ; ou encore rechercher les coefficients du critère linéaire $\left(\mathrm{Y}_{\mathrm{i}}^{*}\right)$ maximisant l'espérance du progrès génétique. Le choix de la régression linéaire $\tilde{Y}_{i}$ peut être apprécié comme un compromis entre les 2 critères $\bar{Y}_{i}$ et $Y_{i}^{*}$, le $1^{\text {er }}$ étant aussi simple mais moins efficace que $\bar{Y}_{i}$, le $2^{\mathrm{e}}$ optimal dans la classe des prédicteurs linéaires mais difficile à déterminer explicitement.

L'hypothèse de normalité joue de toute évidence un rôle fondamental dans l'expression des critères, justifiant en particulier la recherche d'une forme linéaire de l'indice de sélection. Dans d'autres situations (caractères discrets, par exemple) il faudrait donc reprendre entièrement l'analyse.

Nous nous sommes placés dans le cas de la sélection des mâles sur descendance et sous la restriction de non apparentement des candidats à la sélection, de leurs conjointes et des éventuels pères extérieurs inconnus. On peut bien entendu généraliser ce type d'analyse à l'indexation de reproducteurs sur la base d'informations mesurées sur divers types d'apparentés et dans le cas où l'ensemble des animaux candidats et mesurés sont liés, avec incertitude, par des relations d'apparentement.

Reçu le 27 janvier 1983.

Accepté le 10 janvier 1984.

\section{Références bibliographiques}

BONAITI B., 1975. Influence du taux d'erreur dans les filiations paternelles sur la valeur de la sélection sur descendance chez les ovins. Ires Journées de la Recherche Ovine et Caprine, Paris, 2-4 décembre 1975, SPEOC-ITOVIC, 166-172.

Cochran W.G., 1951. Improvement by means of selection. Proceedings of the $2^{\text {nd }}$ Berkeley Symposium on Mathematical Statistics and Probability. Newman J., 449-470 (ed.), University of California Press, Berkeley.

GofFinet B., ElSEN J.M., 1984. Critère optimal de sélection : quelques résultats généraux. Génét. Sél. Evol., 16, 307-318. 